\title{
Case report of brachycephalic obstructive airway syndrome in brachycephalic dogs from Veterinary Specialist Service Hospital, Australia
}

\author{
Phuc N. Le ${ }^{1}$, Thong Q. Le ${ }^{1 *}, \&$ Philip Moses ${ }^{2}$ \\ ${ }^{1}$ Faculty of Animal Science and Veterinary Medicine, Nong Lam University, Ho Chi Minh City \\ ${ }^{2}$ Veterinary Specialist Service Hospital, Underwood, Queensland, Australia
}

ARTICLE INFO
Research Paper
Received: March 04, 2020
Revised: May 15, 2020
Accepted: June 19, 2020
Keywords
Brachycephalic syndrome
BOAS
Brachycephalic dogs
Le Quang Thong
Email: lqthong@hcmuaf.edu.vn
* Corresponding author

Cited as: Le, P. N., Le, T. Q., \& Philip Moses. (2020). Case report of brachycephalic obstructive airway syndrome in brachycephalic dogs from Veterinary Specialist Service Hospital, Australia. The Journal of Agriculture and Development 19(3), 28-38.

\section{Introduction}

Congenitally, brachycephalic dogs are characterized with a shortened head, which is brachycephalic syndrome also known as brachycephalic conformation of the skull (Bjorling et al., 2000). The syndrome consists of anatomic abnormalities including stenotic nares, tortuous turbinates, caudally displaced maxillae, elongated soft palate, everted laryngeal saccules, and hypoplastic trachea (Ackerman, 1999; Koch et al., 2003). The abnormal skull's anatomy narrows the lumen of upper respiratory tract, thus lead to asphyxiation and collapse during excitement, hot weather or exercises (Koch et al., 2003; Packer et al., 2012). Moreover, the displaced maxillae and the elongated soft palate interfere with laryngeal functions resulting in respiratory stridor, open-mouth breathing, inspiratory dyspnea, exercise intolerance, noisy breathing, suffocating and coughing (Ackerman, 1999; Dupre, 2008). According to skull measurements, the typical brachycephalic breeds include Chihuahua, Bulldog, King Charles Spaniel, Pug, Boston Terrier, Maltese, Pekingese, Miniature Pinscher, Shih Tzu, Yorkshire Terrier, and Boxer, 
Lhasa Apso, Shar Pei (Koch et al., 2003). In recent years, the brachycephalic dogs have become the popular breeds in many countries as well as in Vietnam, which inevitably leads to an increase in BOAS cases (Best et al., 2016).

Treatment-wise, hypoplastic trachea, foreshortened maxillae, and narrow rima glottidis are unchangeable. For the case of tortuous turbinate, the surgery can remove a little piece in turbinate to make the airway more ventilated but it is dangerous and expensive with long surgical time. However, the surgery can also affect the patient's olfactory ability, therefore, it is often deemed unnecessary. For the remaining anomalies, there are safer procedures to relieve the symptoms of BOAS, which include trimming the stenotic nares, resecting the elongated soft palate, removal of the everted laryngeal saccules, and removal of the tonsils (depending on the specific situation).

In general, the surgery of stenotic nares includes nares amputation, wedge resection (alarplasty) and alapexy (Fossum, 2013). Specifically, in wedge resection, stenotic nares are resected easily by cutting the $\mathrm{V}$-shaped section of the nares with the No.11 scalpel blade. Wedge resection is less surgical time than alapexy, less incisional bleeding than amputation. However, this procedure can be failed if flaccidity of the cartilage occurs, mobility of the dorsolateral cartilage increases, depigmentation or asymmetrical nose presents. Resection of elongated soft palate is normally performed using Metzenbaum scissor. Electrosurgery can also be used instead; however, may cause swelling in post-operative care. In some cases that tonsils inflamed or obstruct the airway, tonsils can be removed by Metzenbaum scissor or scalpel blade. Then, at the base of the everted tissue, using the tip of a long-handled, curved Metzenbaum scissors transects the everted laryngeal saccules.

To determine which surgical procedures are suitable for the patients, clinical examination and diagnosis are conducted. In clinical examination, the stenotic nares, the size of the trachea and the obstructive inspiratory dyspnea with stertor can be determined by observation and palpation. After that, diagnostic radiography and bronchoscopy rule out abnormal respiratory and cardiology diseases. The surgical procedure should be performed as soon as possible for dog that is above 4 months of age since the nasal tissues are mature enough to hold sutures.

\section{Materials and Methods}

\subsection{Case 1}

\subsubsection{History}

Dog 1 was a six-year-three-month-old male neutered Staffordshire bull terrier breed dog. Noisy breathing was observed by owner; however it became worse during the past year, together with exercise intolerance, heat intolerance, and respiratory struggle during excitement. The dog would occasionally regurgitate white foam, despite eating and drinking normally without coughing or sneezing, or changing in bark.

\subsubsection{Clinical examination}

Clinical examination revealed lean body condition, noticeable upper respiratory noise, pink mucous membrane, good airflow through both nostrils; elongated soft palate was observed. Based on the result, the dog was diagnosed with signs of BOAS. The obstruction of airway was likely due to elongated soft palate and small probability of laryngeal paralysis, with recommendation for BOAS surgery and arytenoid lateralization.

\subsubsection{Laboratory test}

The result of the blood test was $37 \%$ and 70 for packed cell volume and total protein respectively, which is ordinary according to the normal range of PCV (37-55\%) and normal range of TP (55$75)$. This test is quick, easy and it is a common preoperative test because it gives information of the patient's status about anemia, blood protein, hydration status.

\subsubsection{Radiograph}

Findings in thoracic radiographs were unremarkable (Figure 1). The result of imaging diagnosis showed that there were no cardiology and respiratory problems such as heart base tumor, nasopharyngeal, laryngeal, tracheal masses; and secondary changes to the lungs, such as bronchiectasis and probable hypoplastic trachea.

\subsubsection{Bronchoscopy}

The upper airway was examined with bronchoscopy showing the arytenoids moved bilater- 


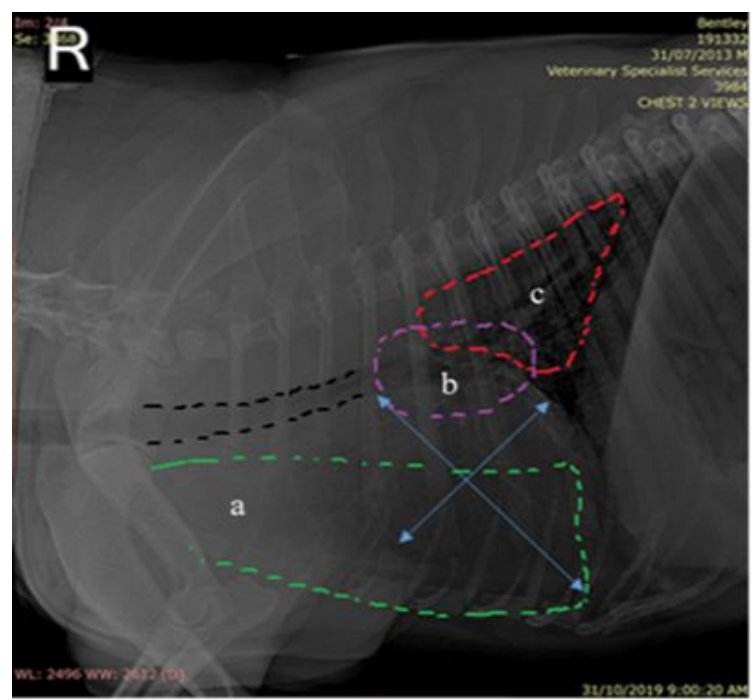

Figure 1. The right lateral thorax radiography (Dog 1). Ventral side (a), Perihilar (b), Dorsocaudal (c) and diffuse of lungs assessed for bronchopneumonia, especially aspiration pneumonia, pulmonary edeama, pulmonary haemorrhage. Vertebral heart size $(\mathrm{VHS})=8(<10.7)$, heart size was normal. Tracheal hypoplasia was rejected (black line) and no hiatus hernia, no abnormalities in vertebral body.

ally, hence arytenoid lateralization was deemed unnecessary.

\subsubsection{Surgery}

The surgical procedure included general anesthesia, upper airway exam, thoracic radiographs and elongated soft palate resection. The patient was then pre-oxygenated for 5-10 minutes and slowly induced with Alfaxalone $30 \mathrm{mg} I \mathrm{~V}$, and intubated with a cuffed ET tube. Circulation, heart rate, oxygenation, ventilation, blood pressure values including systolic arterial pressure, diastolic arterial pressure, mean arterial pressure and the anesthetic maintenance was updated every five minutes using intraoperative monitoring system.

First, the patient was placed in sternal recumbency with the mouth fully opened and the chin was not allowed to have contact with the table's surface. Next, the mucosal surfaces should not be scrubbed to protect from irritation and edema; the endotracheal tube was secured to the lower jaw ensuring free access to the soft palate. Then, the surgeon began to scrub and prepared the soft palate kit when everything was in position (Figure 2).

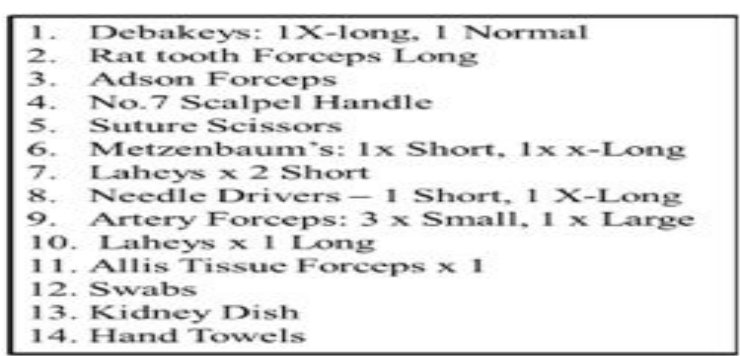

Figure 2. List of surgical instruments for BOAS surgery.

For elongated soft palate resection, the resection was done with scissors, the surgeon placed stay suture of 3-0 Monocryl at the proposed site of resection. Next, the surgeon resected one third of the soft palate with Metzenbaum scissor, then apposed the mucosa with 3-0 Monocryl simple continuous suture pattern; the procedure was then repeatedly continued between excision and suturing until the resection was completed (Figure 3). During the resection, there was mild hemorrhage, which was put under control by tying a swab to a thread and placed the swab in the surgical area.

\subsubsection{Post-operative care}

After surgery, the patient was moved to the Pet intensive care unit (PICU) to recover overnight with close monitoring. Postoperative care included the late extubation, analgesic protocol, nasal oxygen supplementation, close monitoring of the breathing. Upper airway obstruction in post-surgery was concerned due to inflammation and swelling. After surgery, Medetomidine was needed for anxiety, with transition to Tramadol Oral the next day. Post-surgery medications: Methadone $0.1-0.2 \mathrm{mg} / \mathrm{kg} \mathrm{SC}$ and Medetomidine CRI at $1 \mathrm{\mu g} / \mathrm{kg} / \mathrm{h}$. After 2 days in the PICU, the dog swallowed the food trial and was discharged.

\subsection{Case 2}

\subsubsection{History}

A one-year five-month-old female French bulldog was examined for a history of some upper respiratory noise and coughing. The dog ate quickly and sometimes slightly choked on food. Otherwhile, the dog had not shown any significant respiratory difficulty. 

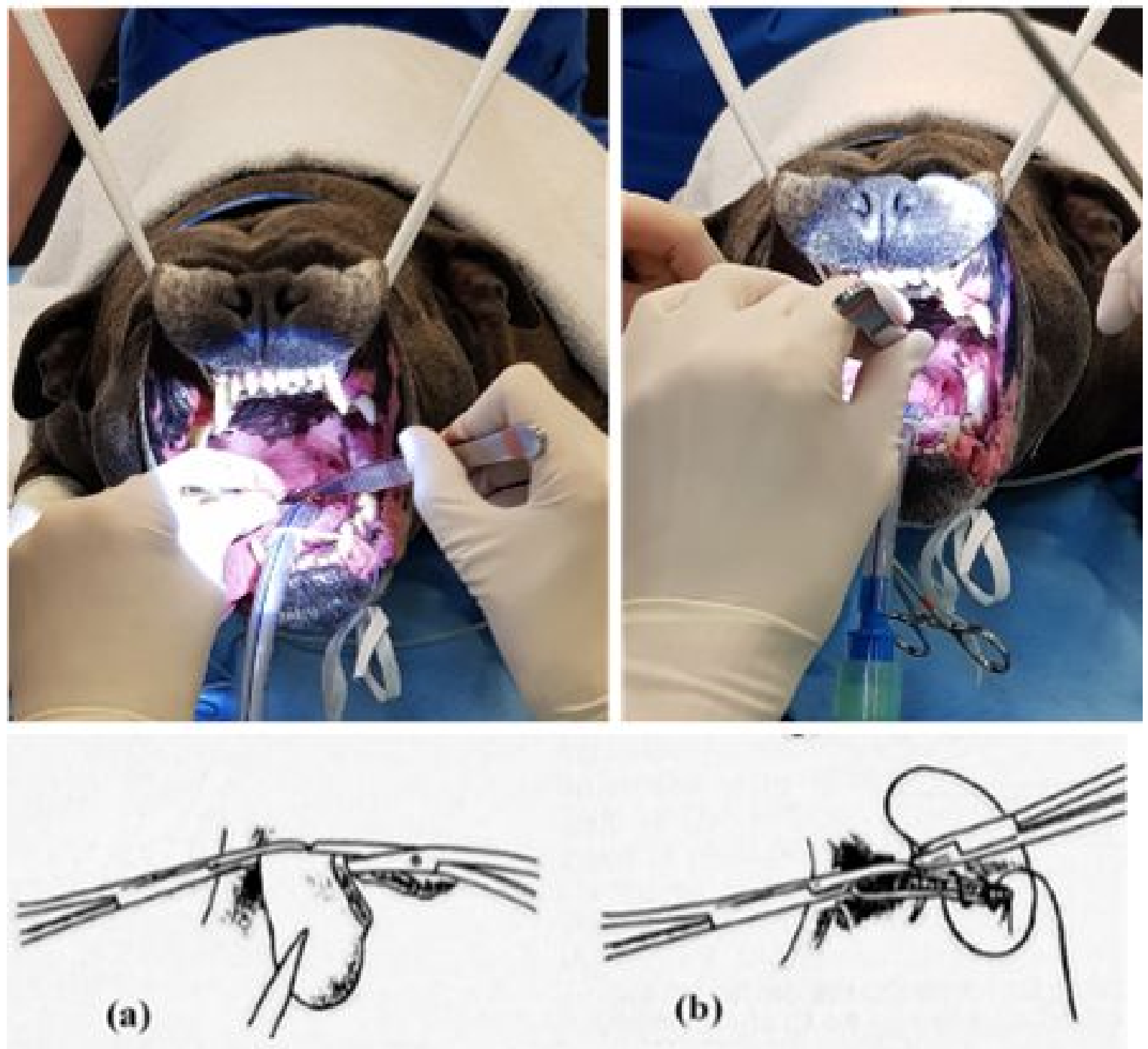

(c)

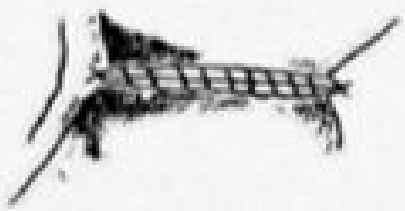

Figure 3. Elongated soft plate resection (Dog 1). (a) Transected one-third of the palate, then (b) apposed the mucosa with sutures. (c) Continue alternating excision and suturing until the resection was completed (Bjorling et al., 2000).

\subsubsection{Clinical examination}

On initial physical examination, the dog possessed congenital traits of brachycephalic breeds. Due to observational heavy open-mouth breathing, respiratory stridor, and difficulty sleeping, elongated soft palate was considered as a main cause. The dog nostrils were congenital stenotic. Thoracic auscultation showed normal cardiac and bronchovesicular sounds, however there was a slight upper airway noise. Based on clinical examination, the first diagnosis was BOAS and surgery therapy was recommended. 


\subsubsection{Laboratory test}

The result of $\mathrm{PCV} / \mathrm{TP}$ was normal at $45 / 74$ based on normal range.

\subsubsection{Radiograph}

The radiograph was conducted to detect other diseases; especially aspiration pneumonia because of the dog's eating routine. The findings were unremarkable (Figure 4).

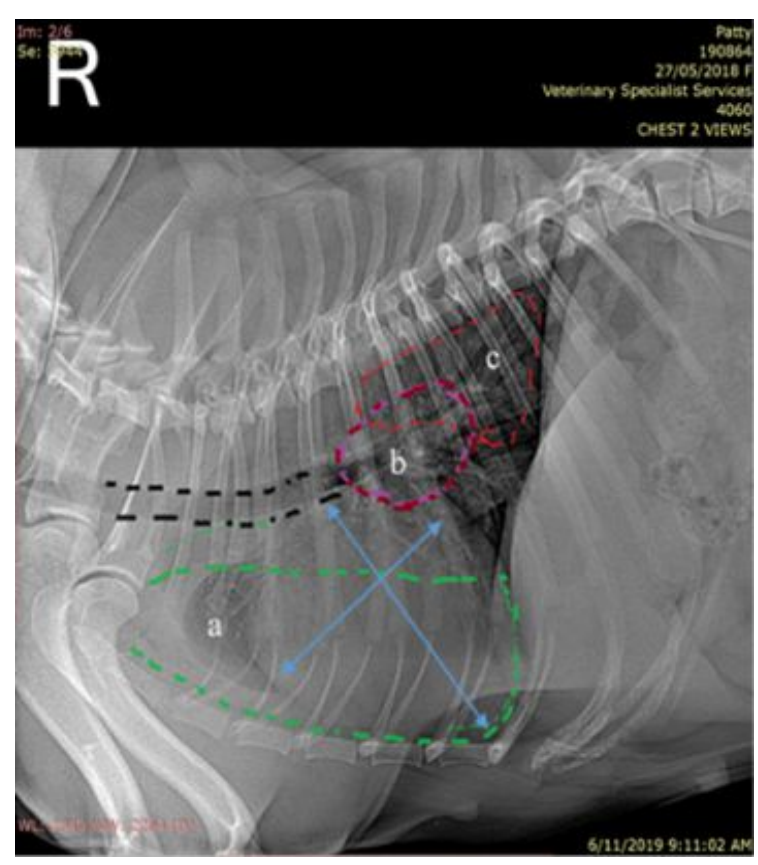

Figure 4. The right lateral thorax radiography (Dog 2). No hiatus hernia, no abnormalities in vertebra, lungs, trachea. Heart size was normal (VHS $=9<$ $10,7)$.

\subsubsection{Bronchoscopy}

During bronchoscopy, laryngeal paralysis was ruled out; no laryngeal saccules were observed. Main bronchus and secondary bronchi were observed no abnormalities (Figure 5).

\subsubsection{Surgery}

The treatment regimen included the resection of elongated soft palate and the resection of stenotic nares.

During soft palate resection surgery, a stay suture of 4-0 Monosyn was placed in the caudal midpoint of the soft palate, allowing it to be pulled rostrally. Two additional stay sutures were placed to either side of the soft palate to mark the intended line of resection, level with the caudal tonsillar crypts. The left side of the soft palate was cut with Metzenbaum scissors, then 4-0 Monosyn simple continuous pattern was used to appose the nasal and oral mucosal cut edges of the soft palate. The process was repeated to remove the remaining soft palate. Next, for stenotic nares resection, wedge resection was performed to permanently enlarge the external nares. Using an 11 scalpel blade, a triangular wedge of tissue was removed from the lateral aspect of the nares. Closure was achieved with 4-0 Monocryl, absorbable sutures placed in a simple interrupted pattern (Figure 6).

\subsubsection{Post-operative care}

After surgery, $\operatorname{dog} 2$ was moved to the PICU for recovery. IV Hartmann's was maintained, meloxicam $1.5 \mathrm{mg} / \mathrm{mL}$ was used once per day when eating to reduce postoperative inflammation and pain; cephalothin $1 \mathrm{~g} / \mathrm{mL}$ IV was supplied. Day 2 in PICU, the patient was stable and swallowed the food trial. The patient was discharged and went back home with administered meloxicam 1.5 $\mathrm{mg} / \mathrm{mL}$ once per day.

\subsection{Case 3}

\subsubsection{History}

An eight-month-old male neutered French bulldog was examined for a history of upper respiratory noise with decreased exercise tolerance. Similar to case 1 , the dog struggled on hot days and during excitement. The main presenting problem was mild upper respiratory stertor.

\subsubsection{Clinical examination}

The dog possessed anatomic abnormality of brachycephalic dog breeds, false positioning of the teeth, open-mouth breathing. Thoracic auscultation showed normal cardiac and bronchovesicular sounds. Based on clinical examination, the dog was diagnosed with BOAS.

\subsubsection{Laboratory test}

The result of $\mathrm{PCV} / \mathrm{TP}$ was normal at $33 / 70$ based on normal range. 


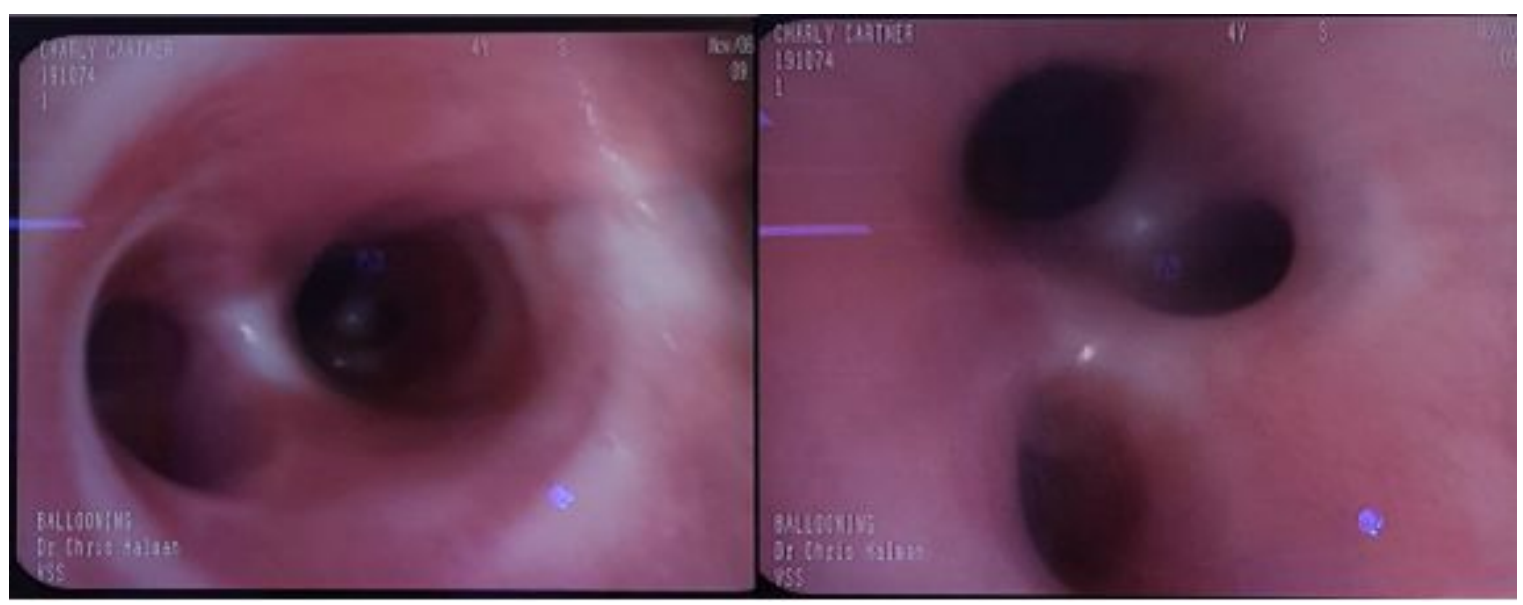

Figure 5. Bronchoscopy (Dog 2). Images of (a) main bronchus and (b) secondary bronchi showed no abnormalities.

\subsubsection{Radiograph}

Imaging diagnosis showed no other problems (Figure 7).

\subsubsection{Bronchoscopy}

Oral examination showed the inflammation of tonsils, moderate elongation of soft palate and bronchoscopy showed erythema around arytenoids; there was no evidence of laryngeal saccule eversion or laryngeal collapse, laryngeal paralysis was ruled out.

\subsubsection{Surgery}

According to diagnosis, the surgeon decided on resection of stenotic nares, resection of elongated soft palate, and tonsils removal. During resection of elongated soft palate, the tip of the soft palate was grasped with allis tissue forceps and a stay suture placed at the site of resection for manipulation. The soft palate was resected approximately one third of the width of the soft palate with Metzenbaum scissors. Closure achieved using 4-0 Monosyn in a simple continuous suture pattern. During resection of stenotic nares, the margin of the nares was grasped with forceps. A $\mathrm{V}$ shaped incision was made medially and the second incision laterally. The wedge of tissue was removed and haemorrhage controlled with pressure. The ventral margin of the nares and the mucocutaneous junction were aligned and sutured closed using 4-0 Monosyn in a simple continuous pattern. For Dog 3, the airway was too small, the tonsils were tonsillitis, obstructed the airway. The surgeon proceeded to remove the protruded tonsils (Figure 8).

\subsubsection{Post-operative care}

After surgery, the dog was moved to the PICU. The dog was noted allergic to Cephalothin, recovered well from anesthesia and surgery, had one regurgitation but was comfortable. The dog continued to be hospitalized in ICU overnight for close monitoring post brachycephalic general anaesthesia and was monitored for risk of aspiration pneumonia. Day 2 in PICU, the dog swallowed food trial well, was bright and alert. Dog 3 showed no regurgitation or emesis, but did have an episode of hypersalivation which was responsive to Maropitant administration which was used the previous night. The patient went back home with Meloxicam $0.1 \mathrm{mg} / \mathrm{kg}$ PO SID when eating.

\subsection{Case 4}

\subsubsection{History}

Dog 4 was an eight-year eight-month-old female Pug with a history of hemivertebrae and ataxia problems. The dog had a surgery to place plate and decompress at two-year old. Based on the hospital transfer record of Dog 4, carprofen injection was used for reducing spinal pain. In the present time, the dog had nasal discharge, dyspnea and bloating; these episodes gradually became more serious and worsened. 

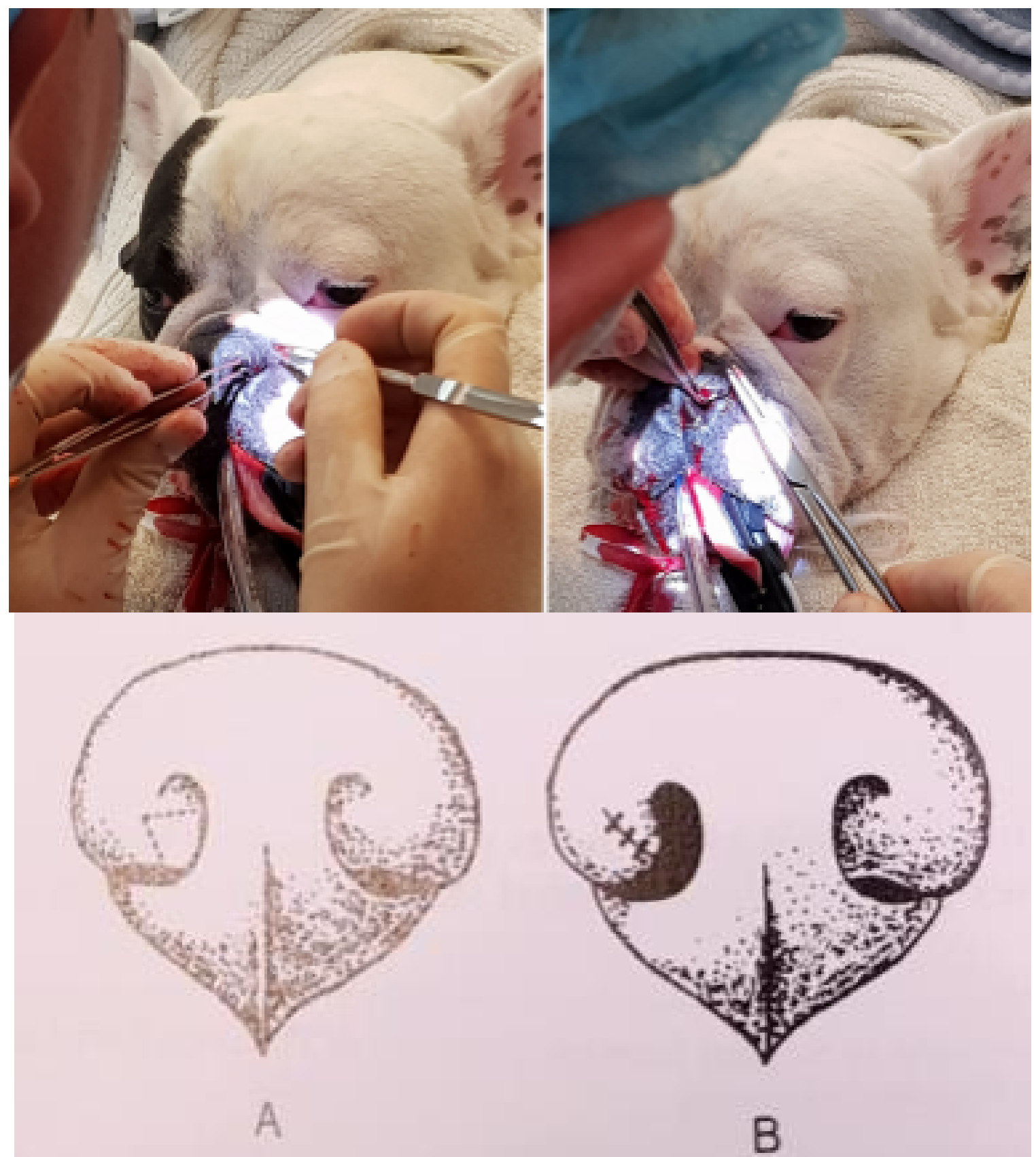

Figure 6. Trimming of stenotic nares (Wedge resection) (Dog 2). (A) Made a V-shaped incision around the forceps with a No. 11 scalpel blade, the first incision was located medially and the second incision laterally. (B) Closed incisions with 4-0 Monocryl, absorbable sutures placed in a simple interrupted pattern (Bjorling et al., 2000).

\subsubsection{Clinical examination}

Tachypnea and moderate respiratory effort were observed. The nares were narrowed, although not severely. Upper respiratory tract noise was increased.
The dog was examined in four days later for assessment of BOAS following an episode of acute dyspnea and bloating before. At this point, clinical signs were consistent with secondary airway obstruction to BOAS. The obstruction was likely associated with elongated soft palate, stenotic 


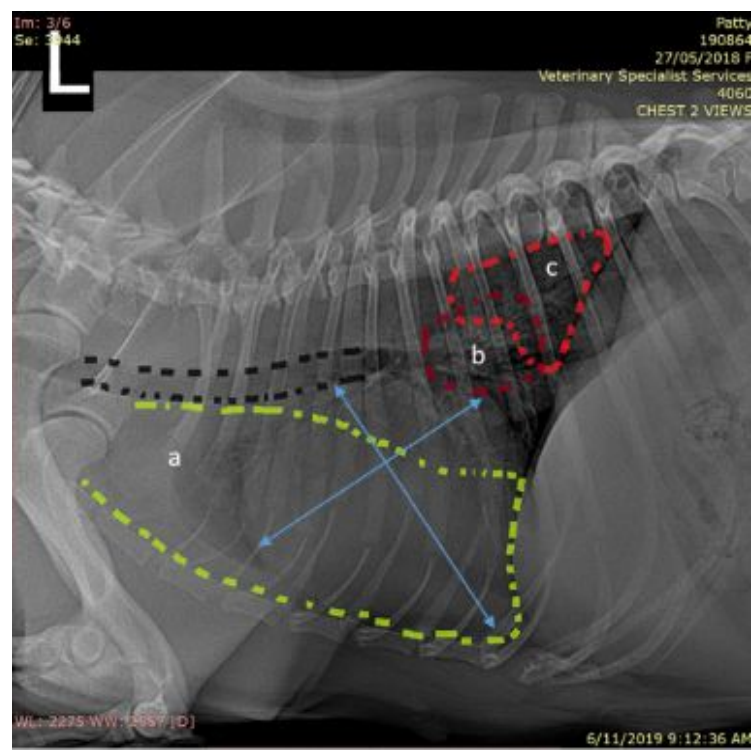

nares and secondary airway abnormalities. Furthermore, the bloating and associated dyspnea noted might also be in part due to gastrointestinal causes, such as a hiatal hernia. Other causes of airway obstruction or respiratory distress could not be ruled out but were investigated prior to surgery.

\subsubsection{Laboratory test}

The PCV/TP test result was normal at 40/70. The ALT in biochemical blood test was higher than normal and this was a caution to use medicine in its treatment (Table 1 ).

Table 1. The result of biochemistry test (Dog 4)

Figure 7. The left lateral thorax radiography (Dog 3 ). Heart size was normal (VHS $=9<10,7$ ). No hiatus hernia, no abnormalities in lungs, trachea.

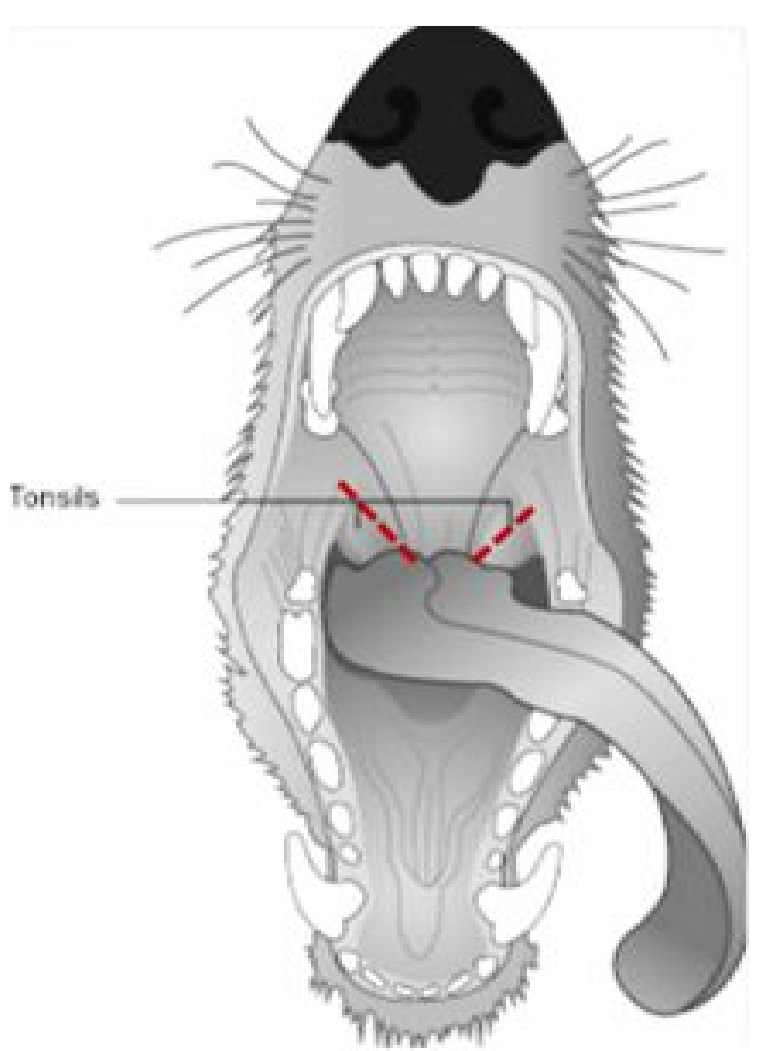

\begin{tabular}{ccccc}
\hline Test & Result & Unit & $\begin{array}{c}\text { Lowest } \\
\text { value }\end{array}$ & $\begin{array}{c}\text { Highest } \\
\text { value }\end{array}$ \\
\hline HEM & 29.0 & & & \\
LIP & 109.0 & & & \\
ICT & 0.0 & & & \\
ALB & 37.0 & $\mathrm{~g} / \mathrm{L}$ & 25.0 & 44.0 \\
$\mathrm{ALP}$ & 33.0 & $\mathrm{U} / \mathrm{L}$ & 20.0 & 150.0 \\
$\mathrm{ALT}$ & $156^{*}$ & $\mathrm{U} / \mathrm{L}$ & 10.0 & 118.0 \\
$\mathrm{AMY}$ & 464.0 & $\mathrm{U} / \mathrm{L}$ & 200.0 & 1200.0 \\
$\mathrm{TBIL}$ & 4.0 & $\mathrm{\mu mol} / \mathrm{L}$ & 2.0 & 10.0 \\
$\mathrm{BUN}$ & 6.1 & $\mathrm{mmol} / \mathrm{L}$ & 2.5 & 8.9 \\
$\mathrm{CA}$ & 2.64 & $\mathrm{mmol} / \mathrm{L}$ & 2.15 & 2.95 \\
$\mathrm{PHOS}$ & 1.27 & $\mathrm{mmol} / \mathrm{L}$ & 0.94 & 2.13 \\
$\mathrm{CRE}$ & 82.0 & $\mathrm{mmol} / \mathrm{L}$ & 27.0 & 124.0 \\
$\mathrm{GLU}$ & 5.1 & $\mathrm{mmol} / \mathrm{L}$ & 3.3 & 6.1 \\
$\mathrm{NA}{ }^{+}$ & 147.0 & $\mathrm{mmol} / \mathrm{L}$ & 138.0 & 160.0 \\
$\mathrm{~K}{ }^{+}$ & 4.6 & $\mathrm{mmol} / \mathrm{L}$ & 3.7 & 5.8 \\
TP & 74.0 & $\mathrm{~g} / \mathrm{L}$ & 54.0 & 82.0 \\
GLOB & 37.0 & $\mathrm{~g} / \mathrm{L}$ & 23.0 & 52.0 \\
\hline \multicolumn{5}{c}{}
\end{tabular}

\subsubsection{Radiograph}

Radiographs revealed some evidence of periimplant lucency which was suggestive of loss of implant stability, but surgical site comfort and palpation was unremarkable (Figure 9). Lung fields were normal with no evidence of aspiration pneumonia.

\subsubsection{Bronchoscopy}

Figure 8. Tonsillectomy (Red lines). Tonsils are removed by forceps and Metzenbaum scissor (Ward \& Hunter, 2009).

Evaluation of the pharynx showed a small central white nodule; different diagnosis was likely an inflammatory nodule. 


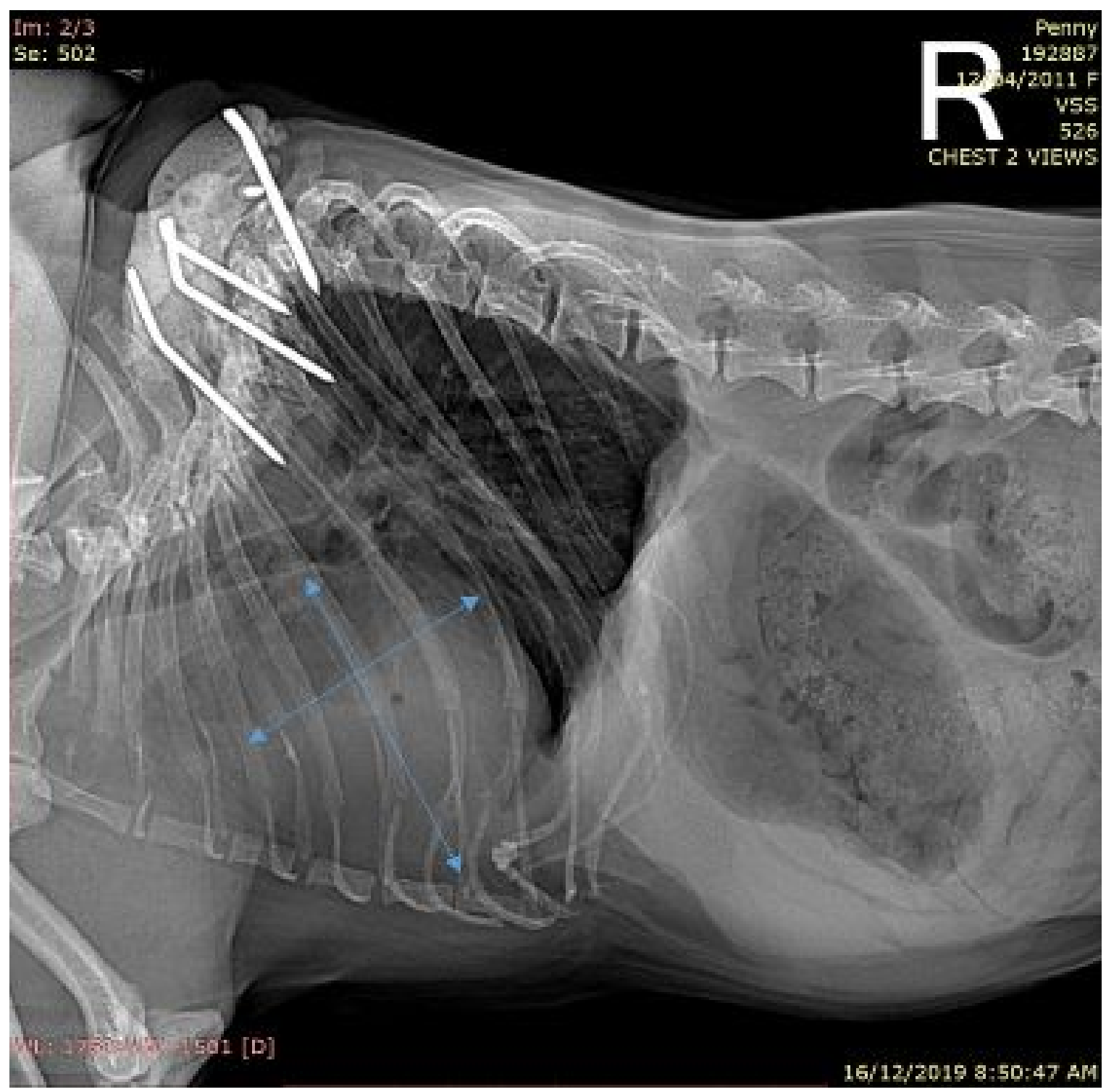

Figure 9. The right lateral thorax radiography (Dog 4). Radiography showed no abnormalities in trachea, lungs, heart, no hiatus hernia. Based on the hospital transfer record, the dog had surgical treatment of hemivertebrae.

\subsubsection{Surgery}

Surgery was recommended for the dog to reduce resistance to inspiration by conducting a combination of alarplasty, soft palate resection and laryngeal sacculectomy if required. First, in staphylectomy, right angle forceps were used for positioning the soft palate and resected using Metzenbaum scissors. Closure achieved using 4-0 Monocryl simple continuous suture pattern. Second, in removal of the laryngeal saccules, the surgeon temporarily extubated Dog 4 . The everted laryngeal saccules were cut off with Metzenbaum scissors (Figure 10). Last, trimming of stenotic nares was performed. A wedge resection of the nares was removed using an 11 scalpel blade. Closure was achieved using 4-0 Monocryl simple interrupted suture pattern.

\subsubsection{Post-operative care}

After surgery, the dog recovered quickly from anaesthetic but then the dog presented dyspnea; thus the oxygen supplement was used. The dog 


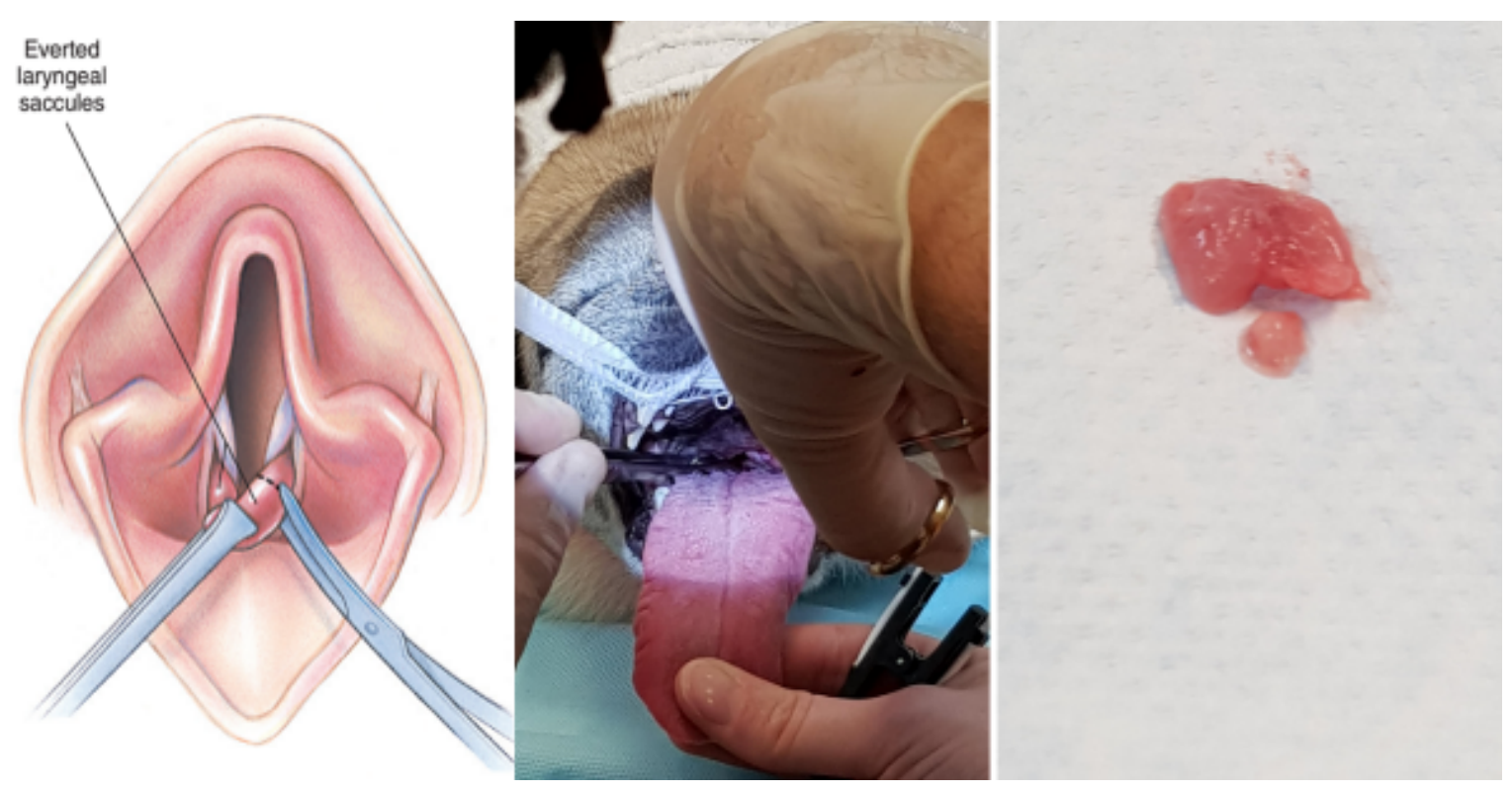

Figure 10. Resection of everted laryngeal saccules (Dog 4). Using Metzenbaum scissor cut off the everted laryngeal saccules (Fossum, 2013).

initially only got inspiratory flow with its mouth held open and tongue out. As recovered from anaesthetic, inspiratory flow was improved but continued to have occasional episodes where the dog retched and moved but then recovered itself. Day 2 in PICU, the dog swallowed the food trial in the morning and then was discharged with omeprazole $10 \mathrm{mg}$ tablet to be given a half twice a day. For the first couple of days, the dog had some difficulty breathing through the nose resulting in self-waking during sleep, causing sleep difficulty. After day 3, the problem was resolved, the dog started to breath well and the gastric reflux seemed to be settled.

\section{Discussion}

Surgery for BOAS should be planned in the morning, after that, observation is recommended for all day. In post-operative care, surgeries complications include bleeding, swelling, edema, wound problems, aspiration pneumonia and even death. Furthermore, post-operative problems can become more complicated, lead to difficulty breathing and respiratory distress (Fossum, 2013). It is treated, depending on severity, with a combination of sedation, oxygen therapy, intubation, temporary or permanent tracheostomy or mechanical ventilation (Holt et al., 1994; O'Dwyner, 2017). Those sort of major com- plications occurred in $10 \%$ of cases, and a small number of dogs do not survive to discharge, typically due to severe aspiration pneumonia.

\section{Conclusions}

BOAS is a set of health problems, mainly in the upper respiratory system, that present predominantly in brachycephalic dog breeds. This is the result of congenital malformation of the skull of such breeds leading to various anatomical abnormalities including stenotic nares, tortuous turbinates, caudally displaced maxillae, elongated soft palate, everted laryngeal saccules, and hypoplastic trachea. These abnormalities consequently cause obstruction in nasal cavity, larynx and/or pharynx. Dogs with BOAS may show signs of having respiratory noises, observable nostril stenosis, eating difficulties, regurgitation, sleep dyspnea, sleep apnea, heat intolerance, exercise intolerance and/or collapsing. Correspondingly, the main focus of BOAS surgeries is to unblock the airway. Surgical procedures may include staphylectomy for the case of elongated soft palate, laryngeal sacculectomy for the case of everted laryngeal saccules, alarplasty for the case of stenotic nares, and tonsillectomy for the case of everted/hypertrophy tonsils. Postoperative results from the case studies show the clear improvement in respiratory health in all discussed 
cases.

\section{References}

Ackerman, L. J. (1999). The genetic connection: a guide to health problems in purebred dogs ( $1^{\text {st }}$ ed.). Colorado, USA: American Animal Hospital Association.

Best, S., Duffin, C., \& Ward, A. (2016). Think twice before getting bulldogs or pugs: Demand for 'flat-faced' canines could damage their health, warn vets. Retrieved May 22, 2020, from https://www.dailymail.co.uk/sciencetech/article3799981/Think-twice-getting-bulldogs-pugs-Vets-saydemand-flat-faced-canines-damage-health.html.

Bjorling, D., McAnulty, J., \& Swainson, S. (2000). Surgically treatable upper respiratory disorders. Veterinary Clinics: Small Animal Practice 30(6), 1227-1251.

Dupre, G. (2008). Brachycephalic syndrome: New knowledge, new treatments. Retrieved January 2, 2020, from https://www.vin.com/apputil/content/defaultadv1.as px? meta $=$ Generic\&pId $=11268 \& i d=3866534$.

Fossum, T. W. (2013). Small animal surgery (4th ed.). Missouri, USA: Elsevier.
Holt, D., \& Brockman, D. (1994). Diagnosis and management of laryngeal disease in the dog and cat. The Veterinary clinics of North America: Small animal practice 24(5), 855-871.

Koch, D. A., Arnold, S., Hubler, M., \& Montavon, P. M. (2003). Brachycephalic syndrome in dogs. Compendium on Continuing Education for The Practising Veterinarian-North American Edition 25(1), 48-55.

O'Dwyner, L. (2017). Anaesthesia for the brachycephalic patient. Retrieved April 28, 2020, from https://www.vin.com/apputil/content/defaultadv1.as px?pId $=20539 \&$ catId $=113426 \&$ id $=8506297 \&$ ind $=401$ \&objTypeID=17.

Packer, R. M. A., Hendricks, A., \& Burn, C. C. (2012). Do dog owners perceive the clinical signs related to conformational inherited disorders as 'normal' for the breed? A potential constraint to improving canine welfare. Animal Welfare-The UFAW Journal 21(1), 81.

Ward, E., \& Hunter, T. (2009). Tonsillitis in dogs. Retrieved July 12, 2009, from https://vcahospitals.com/know-your-pet/tonsillitisin-dogs. 